\title{
Stellar Rotation and Precise Radial Velocities
}

\author{
David F. Gray \\ Astronomy, University of Western Ontario, London, Ontario, Canada
}

\begin{abstract}
This paper focuses on the limitations of establishing rotation rates (to be thought of as differential radial velocities across the surface of the star) and how rotation infringes on our ability to measure precise positions of spectral lines and hence spectroscopic radial velocities.
\end{abstract}

\section{Rotation Rates - Precise Differential Radial Velocities}

You might think that one simply plugs the observations into some standard analysis and out comes the rotation rate. Not so. We typically assume rigid rotation, time invariance, known limb darkening, and an unblemished stellar surface, all of which can be violated. On the bright side, for every new parameter, there is the possibility of measuring it, for example, the inclination of the rotation axis for a differentially rotating star.

Because rotation is usually the dominant line broadener on the hot half of the HR diagram, it can be studied there with relative ease. On the cool half of the HR diagram, macroturbulence (probably mainly granulation seasoned with oscillations) contributes about as much as rotation. Then the central issue becomes how well we can separate the two, and here the resolving power of the spectrograph and the signal-to-noise ratio of the observations are crucial factors.

\section{The Ambiguity of a Non-uniform Disk}

\subsection{Continuum Darkening}

With standard continuum limb darkening, the more darkening toward the limb, the less broadening of the lines. The deduced $v \sin i$ ranges $\pm 8 \%$ going from no limb darkening to complete limb darkening. Some people think we can calculate the limb darkening with good precision, but I'm not so sure; we don't even have the temperature distribution(s) in the Sun pinned down terribly well (Ayres 1987; Solanki \& Unruh 1998). Actually, T( $\tau)$ may even vary with the solar cycle. Admittedly this effect in the Sun is small, but then the strength of magnetic cycles in other stars can be vastly stronger, so why shouldn't variations in temperature distribution, and hence limb darkening, also be stronger? We might be better off on the hot side of the Granulation Boundary (Gray \& Nagel 1988), since we don't see magnetic cycles there, but then there are photospheric velocity fields in these hotter stars that are still a mystery (e.g., Gray 1989a). If rotation is less than $\sim 3 / 4$ the break-up rate, specifying the limb-darkening coefficient $\epsilon$ to \pm 0.06 corresponds to an ambiguity of $1 \%$ in $v \sin i$. 
Rotation closer to the break-up rate causes gravity darkening. Because the specific intensity is proportional to the local effective gravity, the star has a darker girdle around its bulging middle. The fraction of the apparent stellar disk occupied by the darker regions depends on the inclination, so another variable is introduced. The effect of gravity darkening on rotation measurement was studied in some detail in past years, e.g., Sonneborn \& Collins (1977), Slettebak, Kuzma, \& Collins (1980), Stoeckley \& Buscombe (1987), but I have not seen many recent applications.

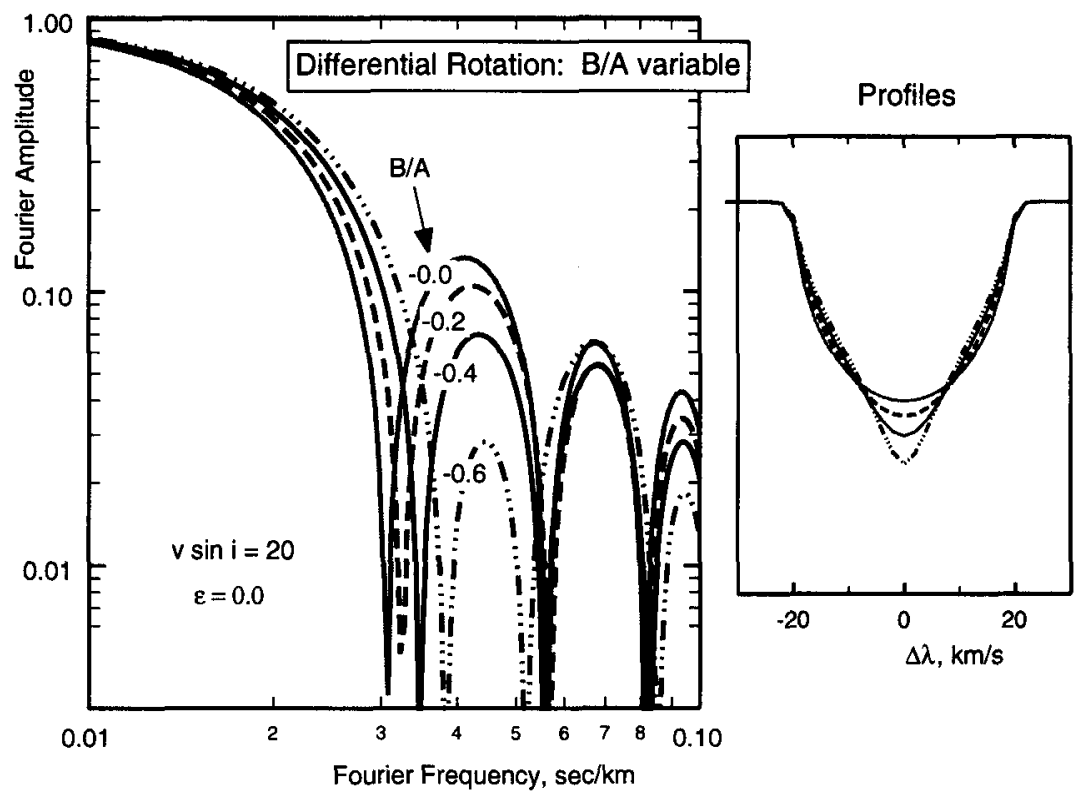

Figure 1. The amplitude of the first sidelobe decreases rapidly with increasing differential rotation in the solar sense (equator fastest)

\subsection{Profile Variations Across the Disk}

Closely related to continuum limb darkening is the center-to-limb variation of the line profile. In the usual analysis, the intrinsic line profile plays no role at all (Gray 1988; Dravins, Lindegren \& Torkelsson 1990). One argues that the thermal broadening is negligible compared to the broadening of rotation and macroturbulence. This seems to be a good approximation in the majority of cases, but in certain situations, it fails badly. For example, when we view a rapidly rotating star pole-on, gravity darkening introduces a significant drop in temperature toward the limb. Spectral lines that are particularly sensitive to temperature respond by showing a dramatic variation with limb distance. Now variation in line strength acts as a weighting factor in the disk integration, and 
the normal limb darkening also amounts to a weighting, so each spectral line has its own effective limb darkening. If the spectral line in question strengthens toward the limb, it effectively has limb brightening.

Vega $(\mathrm{A} 0 \mathrm{~V})$ is a candidate for this situation according to Gulliver, Hill, \& Adelman (1994), where temperature sensitive lines show a distinctly squared-off appearance or even a central reversal. From an analysis of Fe I $\lambda 4522, \mathrm{Fe} I$ $\lambda 4528$, and $\mathrm{Ti}$ II $\lambda 4529$, they find $v \sin i=21.8 \mathrm{~km} / \mathrm{s}$ (equatorial rate is 245 $\mathrm{km} / \mathrm{s}$ ) for their best interpretation, compared to $v \sin i=23.7 \mathrm{~km} / \mathrm{s}$ for the "standard analysis," an error of $9 \%$.



Figure 2. Differential rotation increasing pole-ward results in stronger sidelobes corresponding to more square profiles

\section{The Ambiguity of Differential Rotation}

\subsection{Surface Differential Rotation - Latitude Variations}

Rotation profiles and their Fourier transforms depend on the amount of surface differential rotation, the inclination of the rotation axis, and the relative size of the macroturbulence. Solar differential rotation is quite complex, with different indicators showing different latitude dependence, and there is even evidence for variation during the magnetic cycle (Snodgrass 1992; Howard 1992; Komm et al. 1992). A typical expression describing the solar rotation is, 


$$
v(l)=v_{e q}\left[1+B \sin ^{2} l+C \sin ^{4} l\right]
$$

in which $v_{e q}$ is the equatorial velocity, $l$ is the latitude, and $\mathrm{B}=-0.14, \mathrm{C}=-0.15$.

Evidence for differential rotation in other stars comes from rotational mapping and from variation of periods of rotational modulation over several years (e.g., Bruning 1991; Gray \& Baliunas 1997; Donahue, Saar, \& Baliunas 1996), but not yet from the shapes of spectral lines. Figs. 1 and 2 illustrate shape changes. For equator-fastest cases, the first sidelobe declines (see also Gray 1977, 1982, 1988; Bruning 1981; Garcia-Alegre, Vázques, \& Wöhl 1982). If the differential effect is opposite the solar variety, then the profiles are generally "squared up" and all the sidelobes are stronger. But the figures can be slightly misleading because the rotation rate has to be at least a few times larger than the macroturbulence dispersion, $\zeta_{R T}$, to make the second sidelobe visible against the 'filtering' of macroturbulence. The same statement holds for the resolving power of the spectrograph. Without the second sidelobe we cannot be sure that we have the signature of differential rotation.

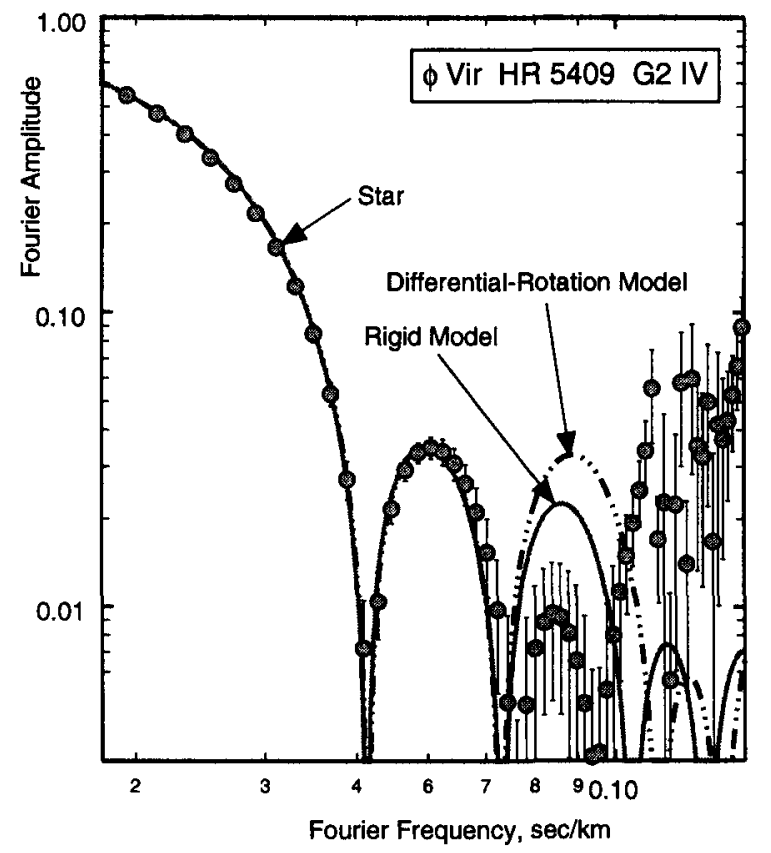

Figure 3. The observations of $\phi$ Vir are equally well matched by a rigid-rotation model having $v \sin i=14.6 \mathrm{~km} / \mathrm{s}$ and a differentiallyrotating model with equatorial velocity of $13.6 \mathrm{~km} / \mathrm{s}$ and $\mathrm{B}=+0.50$, $\mathrm{C}=0.0$.

Typical observations are shown in Fig. 3. The main lobe and the first sidelobe are passably well matched by the two models shown there. Because the 
Fourier transform of the instrumental profile falls to smaller and smaller values toward higher frequencies, the noise is magnified and grows rapidly toward the right side of the panel. Specifically, the noise is large for the second sidelobe, making it untrustworthy, but this is where the two models might have been distinguished. There is nothing unreasonable about any of these numbers; they're just ambiguous.

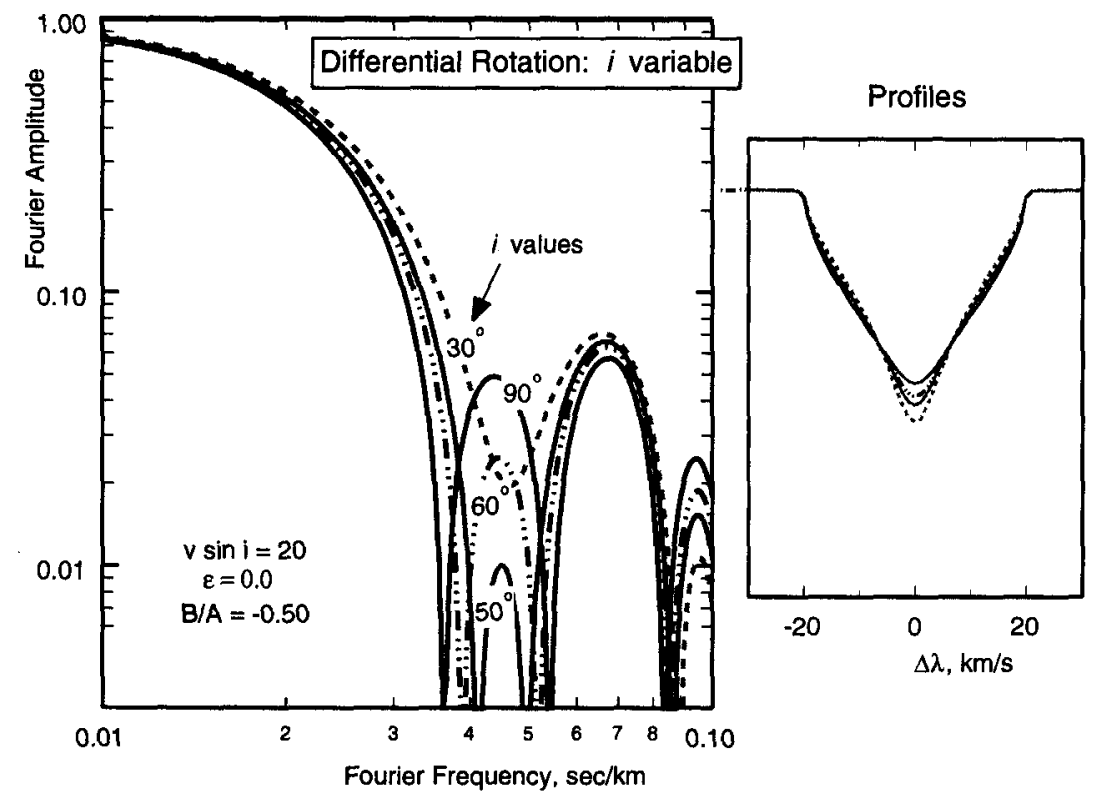

Figure 4. This model has rotation increasing toward the poles at about twice the solar gradient. The first sidelobe weakens rapidly as inclination moves pole-ward.

\subsection{Ambiguity of Inclination}

The effect of inclination compounds the ambiguity because it mimics variations in the strength of the differential effect; compare Fig. 4 to Fig. 1. As the models progress from equator-on $\left(i=90^{\circ}\right)$ to more pole-on orientations, the first sidelobe declines rapidly in size and then disappears altogether. This particular model has about twice the Sun's differential effect. For modest rotation rates, the pointed core and concave sides of the $i=30^{\circ}$ profile (right panel of Fig. 4) might be confused with the shape introduced by Radial-Tangential macroturbulence (see section 4).

When a differentially rotating star is seen near pole-on, a central reversal develops in the line cores. When we do rotational mapping, a central hump maps into a spot at the rotation pole, and quite a few active stars have been interpreted 
to have polar spots. Hatzes et al. (1996) conclude that polar spots do not originate from this artifact, and they are probably right because nearly pole-on orientations would be hard pressed to give the observed rotational modulation.

\subsection{Differential Rotation: Comments}

I have yet to find a certifiable case of differential rotation using spectral line shapes. Stars on the hot side of the Rotation Boundary seem to be rigid rotators. Stars on the cool side of the Rotation Boundary usually rotate too slowly to discern the needed sidelobe signature. Exceptions might be stars in tidally spun-up systems or the odd rapidly rotating single star (Fekel, Moffett, \& Henry 1986; Fekel \& Balachandran 1993), but these candidates usually show profiles distorted by spots (see section 5 ).

You may have noticed that I have not broached the question of arbitrarily complex latitude variations. We might imagine variations that are rapid with latitude, resulting in many small latitude bands, or non-monotonic runs with latitude, or northern/southern hemispheres that are not symmetric, or even flows that are not concentric and therefore have longitude dependence. And then there is height-dependent differential rotation...

\section{The Slow Rotator Problem}

In the mid-1970s, we began to spectroscopically disentangle rotation from macroturbulence for slowly rotating stars. The formulation of the Radial-Tangential macroturbulence model and the introduction of Fourier transforms were the key factors, and there have now been many papers published on rotation rates of cool stars. The central idea is to do disk integrations (Radial-Tangential macroturbulence is not isotropic) that combine Doppler shifts of the rotation with those of macroturbulence. The consistency of the results has been checked statistically against independent information, e.g., Campbell \& Garrison (1985), Gray (1989b).

However, the $v \sin i$ one deduces from these analyses does depend on the assumed macroturbulence model. The Radial-Tangential model is rather simplistic, having radial (vertical) and tangential (horizontal) orientations of the velocity vectors, a Gaussian distribution of velocities of dispersion $\zeta_{R T}$, but no flow velocities of the type one might imagine for rising and falling material in convective motion, and no temperature differences associated with such flows. Errors in $v \sin i$ stemming from failure of the model are probably of the order of a few per cent.

Furthermore, as $v$ sin $i$ falls from $\sim 2 \zeta_{R T}$ to $\zeta_{R T}$, the rotation sidelobe structure decreases rapidly; spectroscopic information on the limb-darkening and differential-rotation essentially disappears. The shape of the main lobe alone is enough to separate rotation from macroturbulence with the assumptions noted above, but does not contain enough information to challenge those assumptions. The internal errors are roughly independent of the rate of rotation. For example, with a typical K0 dwarf having $\zeta_{R T} \sim 2 \mathrm{~km} / \mathrm{s}$, a $v \sin i$ of $4.0 \mathrm{~km} / \mathrm{s}$ is uncertain by $\sim 0.20 \mathrm{~km} / \mathrm{s}$, while a $v \sin i$ of $0.3 \mathrm{~km} / \mathrm{s}$ is uncertain by $\sim 0.15 \mathrm{~km} / \mathrm{s}$. 


\section{Contaminants and Rotation Rates}

Erroneous values of rotation, differential rotation, and radial velocity can arise from unrecognized contaminants: spectroscopic binaries, surface features that produce bumps or asymmetries, oscillations of low degree. Consider the surface feature in Fig. 5. (Of course, if we are lucky and have a whole series of exposures, we would be in the business of rotational mapping instead of simply extracting the rotation rate from the shape of the profiles.) There may be weaker surface features distorting the profile that we cannot so easily identify. If our spectral resolving power is too low, if the spot is spread out in longitude, if the rotation rate is too small to resolve the longitude span of the spot, or if we do not have repeated exposures to show up the time variability, any analysis of the profile for $v \sin i$ will be compromised. Because the spot hump is strongest at line center, we would typically misinterpret the line core as being higher than the true underlying rotation profile, and the fraction of the total line broadening attributed to rotation would be overestimated at the expense of the macroturbulence.

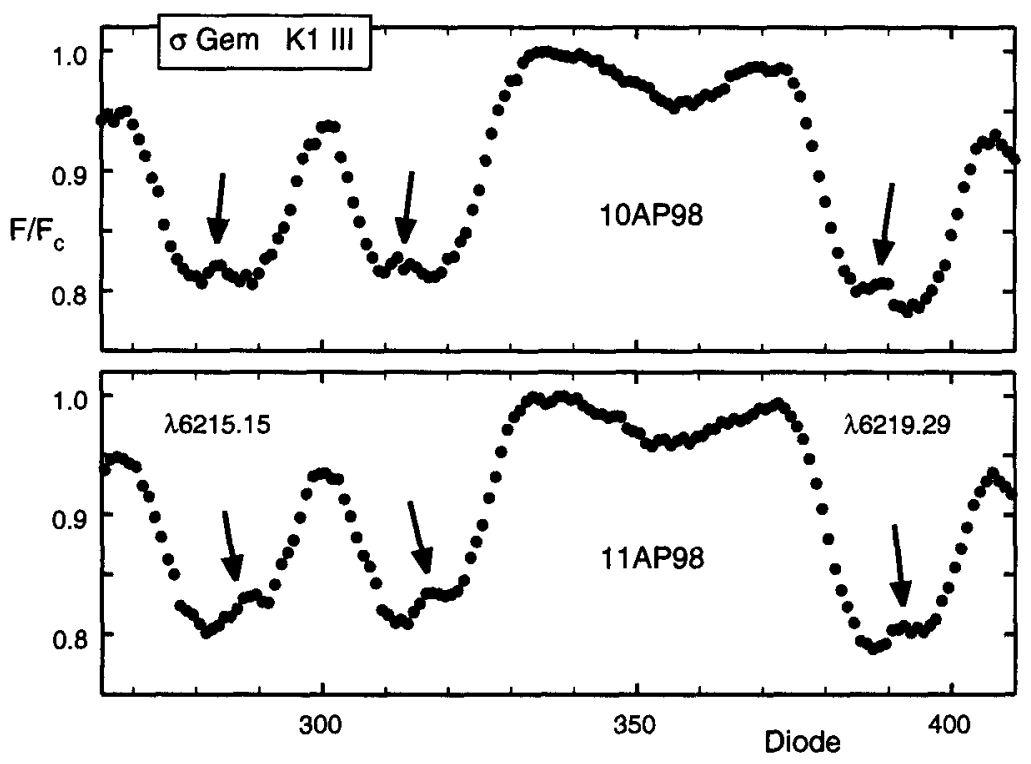

Figure 5. These two exposures on $\sigma$ Gem were taken one day apart. Is it possible to discern the underlying rotation profile? 


\section{Rotational Broadening as a Limiting Factor}

We turn now from the differential radial velocity of rotation to the radial velocity of the whole star and the problem introduced by rotation in measuring the precise positions of spectral lines. Since most of the Doppler shifts we consider are small, we require sharp spectral features to see them. Spectral lines are the only features we have that are sharp, and their sharpness is typically set by the rotation on the hot half of the HR diagram and by rotation combined with macroturbulence on the cool half.

The sharper the spectral line, the better it serves as a marker for a star's radial velocity. Quite generally, velocity errors from photometric noise vary inversely with the slope at any position in the line profile (e.g., Gray 1983) For a pure rotation profile, the slopes vary inversely as the square of the rotational broadening, and therefore uncertainty in the position of the spectral line would increase as the square of $v \sin i$. But as $v \sin i$ goes up, the line will span a greater number of pixels on the detector. Assuming the usual gain with the square root of the number of measurements, the growth in radial-velocity error with rotational broadening is then reduced from the square to the 1.5 power.

Fig. 6 shows data for $\mathrm{G}$ dwarfs published by Saar, Butler, \& Marcy (1998). When rotation is small compared to macroturbulence, it has no sensible effect on the slope of the profile, and therefore there is no dependence of velocity error on rotation. As rotation takes over from macroturbulence, the errors rise in agreement with the predicted 1.5 power dependence. The macroturbulence floor varies systematically with the size of $\zeta_{R T}$ which in turn increases with effective temperature and luminosity.

\section{The Rotation Effect and Systematic Errors}

Rotation and intrinsic asymmetries of spectral lines vary systematically with spectral type, so we must expect systematic radial-velocity errors across the HR diagram. What is not so obvious is that the Doppler shifts of rotation interact with the Doppler shifts of granulation resulting in enhanced asymmetries (the Rotation Effect, Gray 1986, 1988). Calculations for cool stars show blue-shift increasing with rotation rate and with granulation velocities. The net affect is to produce a systematic and spurious negative radial velocity for the star. On the cool half of the HR diagram, the effect increases toward hotter stars (because rotation and granulation velocities do), and if the calculations are to be believed, can amount to a full $\mathrm{km} / \mathrm{s}$ or more. This kind of problem makes it difficult to establish an accurate radial-velocity grid across the HR diagram.

\section{Rotational Modulation Effects}

\subsection{Spots and Patches}

Any surface feature that distorts the shape of a spectral line can be mistaken for a line displacement if the spectral resolution is too poor to show up the details, or if the intrinsic width of the spectral line hides this information. Examples range from dark-spotted cool stars through to chemical blotches on Ap stars. 




Figure 6. Radial velocity errors have a floor set by macroturbulence and rise when rotation dominates the line broadening. Data from Saar et al. (1998).

In practice the ability of a spot to sway the position of the whole line is usually limited to a small fraction of the line width because the spots are typically small. This is indeed the domain of precise radial velocities.

Here spurious velocities will be of both signs and occur equally often. For the casual observer, the "noise" will appear to be larger than expected. But when close monitoring is done, periodic variation will be seen. Complications: multiple spots or continuous gradation in contrast across a large fraction of the stellar disk; the surface feature may have enhanced or repressed velocity fields and magnetic fields in addition to a light deficit or excess.

The spots we see on other stars are usually bigger and stronger than sunspots, and some of them can be relatively stable over many-year time scales (Gray \& Baliunas 1995; Jetsu 1996). Saar \& Donahue (1997) point out that rotation and magnetic effects, spots in particular, should slack off with age, ranging from 200 $\mathrm{m} / \mathrm{s}$ for some Pleiades dwarfs down to less than $10 \mathrm{~m} / \mathrm{s}$ for solar-age stars. See also Toner \& Gray (1988).

\subsection{Non-radial Oscillations}

In slow rotators, non-radial oscillations on the surface of the star produce both centroid shifts and shape distortions of line profiles. The geometry of the os- 
cillations must be simple; too many nodes and the disk integration will reduce them to just another component of macroturbulence (Hatzes 1996). The profilemodulation period is the time it takes the wave to move one wavelength in apparent longitude. I thought we had a good example of this phenomenon with 51 Pegasi (Gray \& Hatzes 1997), but subsequent observations did not bear this out (Gray 1998; Hatzes, Cochran, \& Bakker 1998; Brown et al. 1998). Still, the modeling we presented is indicative of the kind of thing that might result from oscillations: periodic radial-velocity variations of $56 \mathrm{~m} / \mathrm{s}$ as well as $40 \mathrm{~m} / \mathrm{s}$ variations in bisector curvature.

\section{References}

Ayres, T.R. 1987, ApJ, 304, 542

Brown, T.M., Kotak, R., Horner, S.D., Kennelly, E.J., Korzennik, S., \& Noyes, R.W. 1998, ApJ, 494, L85

Bruning, D.H. 1981, ApJ, 248, 274

Bruning, D.H. 1991, PASP, 103, 368

Campbell, B., \& Garrison, R.F. 1985, PASP, 97, 180

Donahue, R.A., Saar, S.H., \& Baliunas, S.L. 1996, ApJ, 466, 384

Dravins, D., Lindegren, L., \& Torkelsson, U. 1990. A\&A, 237, 137

Fekel, F.C., \& Balachandran, S. 1993. ApJ, 403, 708

Fekel, F.C., Moffett, T.M., \& Henry, G.W. 1986, ApJS, 60, 551

Garcia-Alegre, M.C., Vázques, M., \& Wöhl, H. 1982, A\&A, 106, 261

Gray, D.F. 1977, ApJ, 211, 198

Gray, D.F. 1982, ApJ, 258, 201

Gray, D.F. 1983, PASP, 95, 252

Gray, D.F. 1986, PASP, 98, 319

Gray, D.F. 1988, Lectures on Spectral-Line Analysis: F, G, and K Stars, Arva Ontario: The Publisher

Gray, D.F. 1989a, PASP, 101, 832

Gray, D.F. 1989b, PASP, 101, 1126

Gray, D.F. 1998, Nature, 391, 153

Gray, D.F., \& Baliunas, S.L. 1995, ApJ, 441, 436

Gray, D.F., \& Baliunas, S.L. 1997, ApJ, 475, 303

Gray, D.F., \& Hatzes, A.P. 1997, ApJ, 490, 412

Gray, D.F., \& Nagel, T. 1988, ApJ, 341, 421

Gulliver, A.F., Hill, G., \& Adelman, S.J. 1994, ApJ, 429, L81

Hatzes, A.P. 1996, PASP, 108, 839

Hatzes, A.P., Cochran, W.D., \& Bakker, E.J. 1998, Nature, 391, 154

Hatzes, A.P., Vogt, S.S., Ramseyer, T.F., \& Misch, A. 1996, ApJ, 469, 808

Howard, R.F. 1992, in The Solar Cycle, proc. Nat. Solar Obs./Sac. Peak 12th Summer Workshop, K.L. Harvey, 297

Jetsu, L. 1996. A\&A, 314, 153 
Komm, R.W., Howard, R.F., Harvey, J.W., \& Forgach, 1992. in The Solar Cycle, proc. Nat. Solar Obs./Sac. Peak 12th Summer Workshop, K.L. Harvey, 325

Saar, S.H., Butler, R.P., \& Marcy, G.W. 1998, in Cool Stars, Stellar Systems, and the Sun (ASP Conf. Ser., 154), R.A. Donahue \& J.A. Bookbinder, San Francisco: Astron. Soc. Pacific, 1895

Saar, S.H., \& Donahue, R.A. 1997, ApJ, 485, 319

Slettebak, A., Kuzma, T.J., \& Collins, G.W. 1980, ApJ, 242, 171

Snodgrass, H.B. 1992, in The Solar Cycle, proc. Nat. Solar Obs./Sac. Peak 12th Summer Workshop, K.L. Harvey, 205

Solanki, S.K., \& Unruh, Y.C. 1998, A\&A, 329, 747

Sonneborn, G., \& Collins, G.W. 1977, ApJ, 213, 787

Stoeckley, T.R., \& Buscombe, W. 1987, MNRAS, 227, 801

Toner, C.G., \& Gray, D.F. 1988, ApJ, 334, 1008

\section{Discussion}

Hatzes: I have three comments.

1. Years ago I looked into $v \sin i$ effects on RV precision using synthetic spectra and numerical simulations, and found $\delta V_{r} \propto(v \sin i)^{1.1}$. In comparisons with observational data there are two additional effects to consider besides line width: more rapid rotators probably have more spots, and there is a $v \sin i$ amplification effect, where for a given spot size the RV amplitude increases linearly with $v \sin i$.

2. Spots can introduce variations that look like orbits if they are high-latitude spots on a highly inclined star, so that the spots are always in view.

3. On the ambiguity of spots versus differential rotation, a polar spot creates a flat-bottomed profile which looks just like differential rotation with polar acceleration.

Gray: Thanks for your comments.

1. The 1.5-power is "theoretical", being based on the slope and sampling of a typical spectrograph.

2. Yes, a high-latitude spot can roughly mimic orbital motion, but the amplitude of the perturbation will still be smaller when the spot is on the far side of the rotation axis, because its projected area will be smaller. A rather noisy radial-velocity curve might be mistaken as coming from from an eccentric orbit viewed along the major axis, but this mistake would not be made, I think, when the noise is low.

3. Yes, here is a diagram of what you are talking about. Usually the question is whether polar spots are an artifact of differential rotation, but I agree with your earlier assessment that this is unlikely because a central reversal 
from differential rotation comes about only for aspects of view quite close to the pole, and then one would be hard-pressed to explain the rest of the rotational modulation.

Hatzes: It is important for some of our work to derive a very accurate $v \sin i$ for slow rotators and for narrow-line stars. But macroturbulence competes with $v$ $\sin i$ as a dominant broadening mechanism. How accurately can one distinguish between the two? What $\mathrm{S} / \mathrm{N}$ is required, and do you really need to go past that first sidelobe in Fourier space?

Gray: When dealing with slow rotators, where we have to extract rotational broadening when macroturbulence is a major factor, very high signal-to-noise will not help much, and the transform is probably not going to give us much information anyway. Rather the limiting factor is the Radial-Tangential macroturbulence model.

Mayor: We have detected large radial-velocity variations $(30-100 \mathrm{~m} / \mathrm{s})$ for "fast" rotators $(v \sin i=5-10 \mathrm{~km} / \mathrm{s})$ among solar-type stars. In all cases the phase and amplitude are changing on a time scale of a month. These variations cannot be confused with Keplerian motions. We believe that the stability of the phase and amplitude on a span much larger than the lifetime of spots or active regions is a crucial test to identify candidate planets. What is your expectation for the lifetime of the photospheric active regions of solar-type stars with moderate rotation $(v \sin i \sim 5 \mathrm{~km} / \mathrm{s})$ ?

Gray: It's not that simple. In $\epsilon$ Ari, for which we have a decade of H-K modulation, the amplitude changes but the phase does not, i.e., it has an active longitude. Not many studies have been done, but other stars may also be stable. When the phase is the same from year to year, what difference does it make whether it's exactly the same spot or not?

Ecker: A short comment - what about including precession? 